Publ. RIMS, Kyoto Univ.

15 (1979), 719-739

\title{
The Cauchy Problem for Hyperbolic Systems with Variable Multiple Characteristics
}

By

\author{
Hitoshi URYU*
}

\section{§ 1. Hnttraduction}

Recently the Cauchy problem for weakly hyperbolic operators has been studied by many authors. In case of equations with constant multiple characteristics of multiplicity 2, Mizohata and Ohya [7] gave a necessary and sufficient condition for well-posedness of the problem. For the case of higher multiplicity the problem was solved by Flashka-Strange [8] and Chazarain [9] by introducing a generalized Levi's condition. For the case where variable multiple characteristics are concerned, Oleinik [3] obtained a sufficient condition for well-posedness to the equations of the second order. Further, Menikoff [2] extended Oleinik's results to the equations of higher order. Then Ohya [1] simplified Menikoff's proof through extending the method in [7] in a natural manner.

For weakly hyperbolic systems with constant multiple characteristics, either necessary or sufficient conditions for well-posedness are given by Petkov [4], Yamahara [5] and others (c.f. Demay [6]).

In this paper, we shall give a sufficient condition for the Cauchy problem to be well-posed to a first order hyperbolic system with variable multiple characteristics. The proof is done by a method along the ideas of [6] combined with the method of [1].

I should like to express my sincere thanks to Professor S. Irie and Professor T. Kakita for their valuable suggestions and kind encouragement.

\section{§ 2. Statement of $\mathbb{R}$ es nlts}

Consider the Cauchy problem for the first order system of equations,

Communicated by S. Matsuura, July 15, 1977.

* Department of Mathematics, School of Science and Engineering, Waseda University. 
(1) $\quad M[u]=D_{t} u-\sum_{j=1}^{n} A_{j}(x, t) D_{x_{j}} u-B(x, t) u=f(x, t) \quad$ in $\mathbb{R}^{n} \times[0, T]$

$$
u(x, 0)=u_{0}(x)
$$

where $A_{j}(j=1, \cdots, n), B$ are $N \times N$ matrices with entries in $\mathscr{B}\left(\boldsymbol{R}^{n}\right.$ $\times[0, T]), u_{0}, u$ and $f$ are vector valued functions with $N$ components. We denote $D_{t}, D_{x}$ as usual by $-i \partial_{t},-i \partial_{x}$ respectively.

The Cauchy problem for (1) is said to be well-posed for the future $0 \leqq t \leqq T$ in the space $H^{-\infty}\left(\boldsymbol{R}^{n}\right)\left(=H^{-\infty}\right)$ if (i) for each given $u_{0}(x)$ $\in H^{-\infty}$ and $f(x, t) \in \mathscr{B}\left([0, T]: H^{-\infty}\right)$ there exists a unique solution $u$ $\in \mathscr{B}\left([0, T]: H^{-\infty}\right)$ of (1), (ii) linear mapping $\left(u_{0}, f\right) \rightarrow u$ is continuous from $H^{-\infty} \times \mathscr{B}\left([0, T]: H^{-\infty}\right)$ to $\mathscr{B}\left([0, T]: H^{-\infty}\right)$. Here, $H^{-\infty}\left(\mathbb{R}^{n}\right)$ $=\bigcap_{s \in \mathbb{R}^{1}} H^{s}\left(\boldsymbol{R}^{n}\right)$ with the topology: $f_{j}$ converges to 0 in $H^{-\infty}\left(\boldsymbol{R}^{n}\right)$ if and only if for any real $s\left\{f_{j}\right\} \subset H^{s}\left(\mathbb{R}^{n}\right)$ and $f_{j}$ converges to 0 in the space $H^{s}\left(\boldsymbol{R}^{n}\right)$. Since Banach's closed graph theorem (i) implies (ii), we may consider only the existence and uniqueness of solution of (1). Let $\mu_{i}$ $(x, t, \xi)$ for $1 \leqq i \leqq s$ and $\lambda_{j}(x, t, \xi)$ for $1 \leqq j \leqq N-s$ be the whole characteristic roots of the equation of:

$$
\operatorname{det}\left(\tau I-\sum_{j=1}^{n} A_{j}(x, t) \xi_{j}\right)=0 \text { for all }(x, t, \xi) \in \boldsymbol{R}^{n} \times[0, T] \times \mathbb{R}^{n} .
$$

Thus we have the equality

$$
\operatorname{det}\left(\tau I-\sum_{j=1}^{n} A_{j}(x, t) \xi_{j}\right)=\prod_{j=1}^{N-s}\left(\tau-\lambda_{j}\right) \prod_{i=1}^{s}\left(\tau-\mu_{i}\right) .
$$

We assume that for all $i$ and $j$

(i) $\lambda_{j}(x, t, \xi), \mu_{i}(x, t, \xi)$ are real valued smooth functions and

(ii) $\mu_{i} \neq \mu_{j}$ if $i \neq j, \lambda_{i} \neq \lambda_{j}$ if $i \neq j$ and $\mu_{i} \neq \lambda_{j}$ if $i \neq j$.

Now let

$$
\begin{aligned}
& \alpha(x, t, \xi, \tau)=\prod_{j=1}^{N-\delta}\left(\tau-\lambda_{j}\right), \beta(x, t, \xi, \tau)=\prod_{i=1}^{s}\left(\tau-\mu_{i}\right), \\
& P(x, t, \xi, \tau)=\tau I-\prod_{j=1}^{n} A_{j}(x, t) \xi_{j},
\end{aligned}
$$

and $Q(x, t, \xi, \tau)$ be the cofactor matrix of $P$. For convenience we use the notation $x_{0}=t, \xi_{0}=\tau$. By $S^{m}\left(=S_{10}^{m}\right)$ we mean the well-known class of symbols of pseudo-differential operators with respect to $x$. $S^{m}$ is a Frechét space with the semi norms: 


$$
|p|_{l}^{m}=\max _{|\alpha+\beta| \leq l} \sup \left\{\left|D_{\xi}^{\alpha} \partial_{x}^{\beta} p\right|\langle\xi\rangle^{-m+|\alpha|}\right\} .
$$

Let $\widetilde{S}^{m}$ be the set of symbols $p\left(x, x_{0}, \xi, \xi_{0}\right)=\sum_{j=0}^{m} a_{m-j}\left(x, x_{0}, \xi\right) \xi_{0}^{j}$ where $a_{j}\left(x, x_{0}, \xi\right) \in \mathscr{B}\left([0, T]: S^{m-j}\right)$ for $j=0,1, \cdots, m$. We denote by $\mathbb{P}$ a pseudo-differential operator with symbol $P \in \widetilde{S}^{m}$.

Theorem 1. Assume that there exist symbols $X, \widetilde{X} \in \widetilde{S}^{N-2}$ of pseudo-differential operators satisfying the following equalities for some matrices $K_{j}, \widetilde{K}_{j} \in \mathcal{B}\left([0, T]: S^{\mathrm{v}-2}\right) \quad(j=1, \cdots, s)$.

$$
\begin{aligned}
& \text { (I) } \quad\left\{\sum_{j=0}^{n}\left(D_{\xi_{j}} P \partial_{x_{j}} Q-D_{\xi_{j}} \alpha \partial_{x_{j}} \beta I\right)+P X-B Q\right\}\left(x, x_{0}, \xi, \mu_{j}\right)=\left(\mu_{j}-\lambda_{j}\right) K_{j}, \\
& \text { (I) }{ }^{\prime} \quad\left\{\sum_{j=0}^{n}\left(D_{\xi_{j}} Q \partial_{x_{j}} P-D_{\xi_{j}} \alpha \partial_{x_{j}} \beta I\right)+\widetilde{X} P-Q B\right\}\left(x, x_{0}, \xi, \mu_{j}\right)=\left(\mu_{j}-\lambda_{j}\right) \widetilde{K}_{j} .
\end{aligned}
$$

Then the Cauchy problem for (1) is well-posed in the space $H^{-\infty}$ for the future $0 \leqq x_{0} \leqq T$.

Suppose $\mu_{i}(x, 0, \xi) \equiv \lambda_{i}(x, 0, \xi)$ for $i=1, \cdots, s$. Then

Theorem 2. Assume that there exist svmbols $X, \widetilde{X} \in \widetilde{S}^{N-2}$ of pseudo-differential operators satisfying the following equalities for some $K_{j}, \widetilde{K}_{j} \in \mathcal{B}\left([0, T]: S^{N-2}\right) \quad(j=1, \cdots, s)$.

$$
\begin{gathered}
\left\{\sum_{j=0}^{n}\left(D_{\xi_{j}} P \partial_{x_{j}} Q-D_{\xi_{j}} \alpha \partial_{x_{j}} \beta I\right)+P X-B Q\right\}\left(x, x_{0}, \xi, \mu_{j}\right) \\
=\left(\mu_{j}-\lambda_{j}\right) K_{j} / x_{0} \\
\left\{\sum_{j=0}^{n}\left(D_{\xi_{j}} Q \partial_{x_{j}} P-D_{\xi_{j}} \alpha \partial_{x_{j}} \beta I\right)+\widetilde{X} P-Q B\right\}\left(x, x_{0}, \xi, \mu_{j}\right) \\
=\left(\mu_{j}-\lambda_{j}\right) \widetilde{K}_{j} / x_{0} .
\end{gathered}
$$

Then the Cauchy problem for (1) is rell-posed in the space $H^{-\infty}$ for the future $0 \leqq x_{0} \leqq T$.

Remark. It is easy to see that in the above conditions (I), (I)' and (II), (II)' can be replaced $\mu_{j}\left(x, x_{0}, \xi\right)$ by $\lambda_{j}\left(x, x_{0}, \xi\right)$ respectively.

Example of Theorem 1. Consider the Cauchy problem for the 
system

$$
\frac{\partial u}{\partial t}=\left[\begin{array}{cc}
x^{k} & e(x) \\
0 & -x^{k}
\end{array}\right] \frac{\partial u}{\partial x}+\left[\begin{array}{ll}
a & b \\
c & d
\end{array}\right] \text { in } \Omega \times[0, T]
$$

where $\Omega$ is a neighborhood of 0 in $\boldsymbol{R}^{1}$. When we take $X$ $=\left[\begin{array}{cc}0 & 0 \\ 0 & -i\left(a+\lambda_{x}\right)\end{array}\right]$ and $\widetilde{X}=\left[\begin{array}{cc}-i\left(\lambda_{x}-d\right) & 0 \\ 0 & 0\end{array}\right]$ then each left hand side of (I), (I') becomes $\left[\begin{array}{cc}2 a \lambda & -e_{x} \lambda \\ 2 c \lambda & -2 i\left(a+\lambda_{x}\right) \lambda+c e\end{array}\right]$ and $\left[\begin{array}{cc}-(2 a \lambda+c e) & \left(2 b+e_{x}\right) \lambda \\ 0 & 0\end{array}\right]$ respectively where $\lambda=x^{k}$. Hence if $e(x) c(x, t)=x^{k} K(x, t)$ with $K(x, t)$ $\in \mathscr{B}(\Omega \times[0, T])$ the above matrices are divisable by $2 \lambda$. Therefore the Cauchy problem for (2) is well-posed.

Example of Theorem 2. Consider the Cauchy problem for the system

$$
\frac{\partial u}{\partial t}=\left[\begin{array}{cc}
t^{k} & 1 \\
0 & -t^{k}
\end{array}\right] \frac{\partial u}{\partial x}+\left[\begin{array}{ll}
a & b \\
c & d
\end{array}\right] \quad \text { in } \boldsymbol{R}^{1} \times[0, T]
$$

When we take $X=\left[\begin{array}{cc}0 & 0 \\ 0 & -i a\end{array}\right]$ and $\widetilde{X}=\left[\begin{array}{cc}i d & 0 \\ 0 & 0\end{array}\right]$ then each left hand side of (II), (II)' becomes $\left[\begin{array}{lc}2 a \lambda & 0 \\ 2 c \lambda & c+2\left(a \lambda-\lambda_{t}\right)\end{array}\right]$ and $\left[\begin{array}{cc}-2 \lambda_{t}-2 a \lambda-c & -2 b \lambda \\ 0 & 0\end{array}\right]$ respectively where $\lambda=t^{k}$. Hence if $c(x, t)=t^{k-1} K(x, t)$ with $K(x, t)$ $\in \mathscr{B}\left(\boldsymbol{R}^{1} \times[0, T]\right)$ the above matrices are divisable by $2 \lambda / t$. Therefore the Cauchy problem for (3) is well-posed.

\section{$\S 3$. Proof of Theorem $\mathbb{1}$}

We use the following norms.

$\|u\|$ is the $L^{2}$-norm of $u$;

$\|u\|_{s}$ is the Sobolev norm of $u$ i.e. $\|u\|_{s}=\left\|\left(1+|\xi|^{2}\right)^{s / 2} \hat{u}(\xi)\right\|$

$$
\begin{aligned}
& \text { where } \hat{u}(\xi)=\int e^{i x \xi} u(x) d s ; \\
& \left\|D^{m} u\left(x, x_{0}\right)\right\|=\sup _{0 \leqq j \leqq m}\left\|D_{x_{0}}^{j} u\left(x, x_{0}\right)\right\|_{m-j} ;
\end{aligned}
$$

Before we prove Theorem 1 we need following proposition. 
Proposition 3. 1. Let $u \in \mathscr{B}\left([0, T]: H^{l+N-2}\right)$ be a solution of the Cauchy problem for a pseudo differential operator

$$
\begin{aligned}
& (\boldsymbol{\alpha} I+\boldsymbol{C})(\boldsymbol{\beta} I+\mathbb{D}) I u+\mathbb{R}_{N-2} u=f \in \mathscr{B}\left([0, T]: H^{l}\right) \\
& \left(D_{x_{0}}^{j} u\right)(x, 0)=\varphi_{j}(x) \in H^{l+N-j-1} \quad(j=0, \cdots, N-1)
\end{aligned}
$$

where $\sigma(\mathbb{C})=C \in \widetilde{S}^{N-s-1}, \quad \sigma(\mathbb{D})=D \in \widetilde{S}^{s-1}$ and $\sigma\left(\mathbb{R}_{N-2}\right)=R_{N-2} \in \widetilde{S}^{N-2}$. Then ree have the following energy inequalities.

$$
\begin{gathered}
\left\|D^{l+N-2} u\right\| \leqq \text { const. }\left\{\sum_{j=0}^{N-1}\left\|\varphi_{j}\right\|_{l+N-j-1}+\sum_{j=0}^{l-1}\left\|D_{x_{0}}^{j} f(0)\right\|_{l-j-1}\right. \\
\left.\quad+\int_{0}^{x_{0}}\left\|\left(D^{l} f\right)(s)\right\| d s\right\} \\
\text { for } l=0,1, \cdots .
\end{gathered}
$$

The proof is based on the following two lemmas.

Lemma 3.2. Let $\boldsymbol{P}=\mathbb{P}_{m} I+\mathbb{R}_{m-1}$ be a pseudo-differential operator such that $\mathbb{P}_{n}$ is a strictly' hyperbolic operator and $\mathbb{R}_{n-1}$ is an $N \times N$-matrix pseudo-differential operator with symbols in $\widetilde{S}^{m-1}$. Then we have

$$
\begin{aligned}
& \frac{d}{d x_{0}}\left\|D^{l+m-1} u\right\| \leqq \text { const. }\left\{\left\|D^{l+m-1} u\right\|+\left\|D^{l}(\mathbb{P} u)\right\|\right\} \\
& \quad \text { for } l=0,1, \cdots
\end{aligned}
$$

Lemma 3. 3. If $u \in \mathscr{B}\left([0, T]: I^{s}\right)$ is a solution of (4), then we have

$$
\begin{aligned}
& \left\|D_{x_{0}}^{N+l} u(x, 0)\right\|_{s} \leqq \text { const. }\left\{\sum_{j=0}^{N-1}\left\|\varphi_{j}\right\|_{N+l-j+s}+\sum_{i=0}^{l}\left\|\left(D^{i} f\right)(0)\right\|_{l-i+s}\right\} \\
& \text { for } l=0,1, \cdots .
\end{aligned}
$$

Proof of Proposition 3.1. Let us denote $S=\alpha I+\mathbb{C}$ and $\mathbb{R}=\beta I$ $+\mathbb{D}$. Then we have another system of pseudo-differential operators

$$
\begin{aligned}
& \mathbb{R} u=v \\
& S v=f-\mathbb{R}_{N-\mathbf{2}} u .
\end{aligned}
$$


By Lemma 3.2, we immediately have

$$
\begin{aligned}
\frac{d}{d x_{0}}\left\|D^{l^{\prime}+s-1} u\right\| & \leqq \text { const. }\left\{\left\|D^{l^{\prime}+s-1} u\right\|+\left\|D^{l^{\prime}} v\right\|\right\} \\
\frac{d}{d x_{0}}\left\|D^{l+N-s-1} v\right\| & \leqq \text { const. }\left\{\left\|D^{l+N-s-1} v\right\|+\left\|D^{l}\left(f-\boldsymbol{R}_{N-2} u\right)\right\|\right\} \\
& \leqq \text { const. }\left\{\left\|D^{l+N-s-1} v\right\|+\left\|D^{l+N-2} u\right\|+\left\|D^{l} f\right\|\right\}
\end{aligned}
$$

Summing (9) with $l^{\prime}=l+N-s-1$ and (10), we have

$$
\begin{aligned}
& \frac{d}{d x_{0}}\left(\left\|D^{l+N-2} u\right\|+\left\|D^{l+N-s-1} v\right\|\right) \\
& \quad \leqq \text { const. }\left\{\left(\left\|D^{l+N-2} u\right\|+\left\|D^{l+N-s-1} v\right\|\right)+\left\|D^{l} f\right\|\right\} .
\end{aligned}
$$

Then Gronwall's inequality implies

$$
\begin{aligned}
\left\|D^{l+N-2} u\right\| & +\left\|D^{l+N-s-1} v\right\| \leqq \text { const. }\left\{\left\|D^{l+N-2} u(x, 0)\right\|\right. \\
& \left.+\left\|D^{l+N-s-1} v(x, 0)\right\|+\int_{0}^{x_{0}}\left\|D^{l} f(x, s)\right\| d s\right\} .
\end{aligned}
$$

On the other hand by Lemma 3.3 we have

$$
\begin{aligned}
& \left\|D^{l+N-2} u(x, 0)\right\| \leqq \text { const. }\left\{\sum_{j=0}^{N-1}\left\|\varphi_{j}\right\|_{N+l-2-j}+\sum_{i=0}^{l-2}\left\|\left(D^{i} f\right)(x, 0)\right\|_{l-2-i}\right\} \\
& \left\|D^{l+N-s-1} v(x, 0)\right\| \leqq \text { const. }\left\{\sum_{j=0}^{N-1}\left\|\varphi_{j}\right\|_{N+l-1-j}+\sum_{i=0}^{l-1}\left\|\left(D^{i} f\right)(x, 0)\right\|_{l-1-i}\right\} .
\end{aligned}
$$

Now the estimate (5) follows from (11), (12) and (13).

We also need the energy inequalities for the adjoint operator $T^{*}$ of $\boldsymbol{T}=(\boldsymbol{\alpha} I+\boldsymbol{C})(\boldsymbol{\beta} I+\mathbb{D})+\boldsymbol{R}_{N-2}$.

Proposition 3. 4. Let $\mathbb{T}^{*}$ be the adjoint operator of $\mathbb{T}=(\alpha I+\mathbb{C})$ $(\boldsymbol{\beta} I+\mathbb{D})+\mathbb{R}_{N-2}$. Then there exist pseudo-differential operators $\widetilde{\mathbb{C}}, \widetilde{\mathbb{D}}$ and $\widetilde{\boldsymbol{R}}_{N-2}$ with symbols $\sigma(\widetilde{\mathbb{C}}) \in \widetilde{S}^{N-s-1}, \sigma(\widetilde{\mathbb{N}}) \in \widetilde{S}^{s-1}$ and $\sigma\left(\widetilde{\boldsymbol{R}}_{N-2}\right) \in \widetilde{S}^{N-2}$ respectively, satisfying $\mathbb{T}^{*}=(\beta I+\widetilde{\mathbb{D}})(\boldsymbol{\alpha} I+\widetilde{\boldsymbol{C}})+\widetilde{\boldsymbol{R}}_{N-2}$.

Proof. Let us calculate a symbol of $\mathbb{T}$

$$
\sigma(\boldsymbol{T}) \equiv \alpha \beta I+\alpha D+\beta C+\sum_{j=0}^{n} D_{\xi_{j}} \alpha \partial_{x, j} \beta I
$$


where $\equiv$ means modulo functions of $\widetilde{S}^{N-2}$. Then

$$
\begin{gathered}
\sigma\left(\mathbb{T}^{*}\right) \equiv \alpha \beta I+\sum_{j=0}^{n} D_{\varepsilon_{j}} \partial_{x_{j}}(\alpha \beta) I+\alpha^{t} \bar{D}+\beta^{t} \bar{C}+\sum_{j=0}^{n} \overline{D_{\xi_{j}} \alpha \partial_{x_{j}}} \beta I \\
=\alpha \beta I+\sum_{j=0}^{n} \partial_{x_{j}} \alpha D_{\xi_{j}} \beta I+\beta\left\{\sum_{j=0}^{n}\left(D_{\xi_{j}} \partial_{x_{j}} \alpha\right) I+{ }^{t} \bar{C}\right\} \\
+\alpha\left\{\sum_{j=0}^{n}\left(D_{\xi_{j}} \partial_{x_{j}} \beta\right) I+{ }^{t} \bar{D}\right\} .
\end{gathered}
$$

Thus if we take $\bar{C}$ and $\widetilde{D}$ in such a way that

$$
\sigma(\widetilde{\mathbb{C}})=\sum_{j=0}^{n} D_{\xi_{j}} \partial_{x_{j}} \alpha I+{ }^{t} \bar{C} \quad \text { and } \sigma(\widetilde{D})=\sum_{j=0}^{n} D_{\xi_{j}} \partial_{x_{j}} \beta I+{ }^{t} \bar{D}
$$

then we have $\mathbb{T}^{*}=(\boldsymbol{\beta} I+\widetilde{\mathbb{B}})(\boldsymbol{\alpha} I+\widetilde{\boldsymbol{C}})+\widetilde{\mathbb{R}}_{N-2}$.

We define a sesqui-linear form $\langle u, v\rangle$ on $L_{1}^{2}\left([0, T] \times \mathbb{R}^{n}\right)$ by

$$
\left.\langle u, v\rangle=\int_{0}^{T}(u(t), v(t)) d t=\int_{0}^{T} \int_{R^{n}} u(x, t) \overline{v(r, t}\right) d x d t .
$$

Then we have

$$
\begin{gathered}
\langle\mathbb{T} u, v\rangle=\left\langle u, \mathbb{T}^{*} v\right\rangle \text { for } u, v \in C^{\infty}\left([0, T]:\left(C_{0}^{\infty}\left(\mathbb{R}^{n}\right)\right)^{N}\right) \text { with } \\
D_{t}^{j} v(x, T)=0, D_{t}^{j} u(x, 0)=0 \quad(j=0,1, \cdots, N-1) .
\end{gathered}
$$

For the Cauchy problem

$$
\begin{aligned}
& T u=f \\
& D_{i}^{i} u(x, 0)=0 \quad(j=0,1, \cdots, N-1),
\end{aligned}
$$

the energy inequality

$$
\left\|D^{N+l-2} u(t)\right\| \leqq \text { const. } \int_{0}^{t}\left\|D^{l} f(s)\right\| d s
$$

holds by Proposition 3.1. For the adjoint Cauchy problem

$$
\begin{aligned}
& T^{*} v=g \\
& D_{t}^{j} v(x, T)=0 \quad(j=0,1, \cdots, N-1)
\end{aligned}
$$

we also have the energy inequality

$$
\left\|D^{N+l-2} v(t)\right\| \leqq \text { const. } \int_{t}^{T}\left\|D^{l} g(s)\right\| d s
$$


which is derived from Proposition 3. 4 and the proof of Proposition 3.1.

Let us consider a weak form of (16)

$$
\begin{aligned}
& \left\langle u, T^{*} v\right\rangle=\langle f, v\rangle \\
& D_{t}^{j} u(x, 0)=0 \quad(j=0,1, \cdots, N-1)
\end{aligned}
$$

for any $v \in C^{\infty}\left([0, T] ;\left(C_{0}^{\infty}\left(\mathbb{R}^{n}\right)\right)^{N}\right)$ with $D_{t}^{j} v(x, T)=0 \quad(j=0,1, \cdots$, $N-1)$. Then from (19) we obtain

$$
\begin{aligned}
|\langle f, v\rangle| \leqq & \left(\int_{0}^{T}\|f(\tau)\|_{-s}^{2} d \tau\right)^{1 / 2}\left(\int_{0}^{T}\|v(\tau)\|_{s}^{2} d \tau\right)^{1 / 2} \\
\leqq & \operatorname{const.}\left(\int_{0}^{T}\|f(\tau)\|_{-s}^{2} d \tau\right)^{1 / 2} \\
& \times\left(\int_{0}^{T}\left(\int_{0}^{T}\left\|D^{s-N+2}\left(T^{*} v\right)(s)\right\| d s\right)^{2} d \tau\right)^{1 / 2} \\
\leqq & \operatorname{const.}\left(\int_{0}^{T}\|f(\tau)\|_{-s}^{2} d \tau\right)^{1 / 2} \\
& \times\left(\int_{0}^{T}\left\|D^{s-N+2}\left(T^{*} v\right)(\tau)\right\|^{2} d \tau\right)^{1 / 2} .
\end{aligned}
$$

Hence this inequality combined with (17) admit existence and uniqueness of the solution to (16) by Nirenberg's method [10]. Consequently we proved

Theorem 3. 5. The Cauchy problem for (4) is well-posed in the space $H^{-\infty}\left(\boldsymbol{R}^{n}\right)$ for the future $0 \leqq x_{0} \leqq T$.

Now let us prove Theorem 1 .

(i) Existence

Let $v \in \mathscr{B}\left([0, T]: H^{l+N-2}\right)$ be a solution of the Cauchy problem

$$
\begin{aligned}
& \boldsymbol{M}(\boldsymbol{Q}+\boldsymbol{X}) \boldsymbol{v}=f(x, t) \in \mathscr{B}\left([0, T]: H^{l}\right) \quad \text { in } \boldsymbol{R}^{n} \times[0, T] \\
& \left(D_{x_{0}}^{j} v\right)(x, 0)=\varphi_{j}(x) \in H^{l+N-j-1} \quad(j=0, \cdots, N-1) .
\end{aligned}
$$

Then with $\varphi_{N-1}(x)=u_{0}(x)$ and $\varphi_{j}(x)=0(j=0,1, \cdots, N-2), u=(\boldsymbol{Q}+\boldsymbol{X}) v$ will give a solution to our system (1). Therefore it remains only to prove the well-posedness for the Cauchy problem (21). Theorem 3.5 combined with the following lemma gives the existence of a solution to the problem (1). 
Lemrina 3.6. Under the condition (I) there exist symbols of pseudo differential operators $C \in \widetilde{S}^{N-s-1}, D \in \widetilde{S}^{s-1}$ and $R_{N-2} \in \widetilde{S}^{N-2}$ such that

$$
\mathbb{M}(\boldsymbol{Q}+\boldsymbol{X})=(\boldsymbol{\alpha} I+\mathbb{C})(\boldsymbol{\beta} I+\mathbb{D})+\mathbb{R}_{N-2}
$$

Proof of Lemma 3.6. We start with calculation of the symbols of $\mathbb{M}(\boldsymbol{Q}+\boldsymbol{X})$ and $(\boldsymbol{\alpha} I+\boldsymbol{C})(\boldsymbol{\beta} I+\mathbb{D})$. From the formula for symbol of the product of operators it follows

$$
\begin{aligned}
& \sigma(\boldsymbol{M}(\boldsymbol{Q}+\boldsymbol{X})) \equiv \operatorname{det}(P) I+\sum_{j=0}^{n} D_{\xi_{j}} P \partial_{x_{j}} Q-B Q+P X, \\
& \sigma((\alpha I+\mathbb{C})(\beta I+D)) \equiv \alpha \beta I+\sum_{j=0}^{n} D_{\xi_{j}} \alpha \partial_{x_{j}} \beta I+\alpha D+\beta C .
\end{aligned}
$$

Therefore all we want to do is to determine the symbols $C \subseteq S^{\mathrm{v}-s-1}$ and $D \in S^{s-1}$ so that

$$
\sigma(\mathbb{M}(\mathbb{Q}+\mathbb{N})) \equiv \sigma((\alpha I+\mathbb{C})(\beta I+\mathbb{D}))
$$

Then we obtain

$$
\sum_{j=0}^{n}\left\{D_{\xi_{j}} P \partial_{x_{j}} Q-D_{\xi_{j}} \alpha \partial_{x_{j}} \beta I\right\}+P X-B Q=\alpha D+\beta C .
$$

Putting $\xi_{0}=\mu_{j}\left(x, x_{0}, \xi\right)$ into (25) shows

$$
\begin{gathered}
{\left[\sum_{j=0}^{n}\left\{D_{\xi_{j}} P \partial_{x_{j}} Q-D_{\xi_{j}} \alpha \partial_{r_{j}} \beta l\right\}+P X-B Q\right]\left(x, x_{0}, \xi, \mu_{j}\right)} \\
=\prod_{k=1}^{N-s}\left(\mu_{j}-\lambda_{k}\right) D\left(x, x_{0}, \xi, \mu_{j}\right) .
\end{gathered}
$$

By the condition (I) of Theorem, the left hand side is equal to $K_{j}\left(x, x_{0}\right.$, $\xi)\left(\mu_{j}-\lambda_{j}\right)$ where $K_{j} \in \mathscr{B}\left([0, T]: S^{N-2}\right)$. Hence we have

$$
D\left(x, x_{0}, \xi, \iota_{j}\right)=\left[\prod_{\substack{k=1 \\ j \neq k}}^{N-s}\left(\mu_{j}-\lambda_{k}\right)\right]^{-1} K_{j}\left(x, x_{0}, \xi\right) .
$$

Because $D\left(x, x_{0}, \xi, \xi_{0}\right)$ is a polynomial on $\xi_{0}$, we have such a representation as

$$
\begin{aligned}
D\left(x, x_{0}, \xi, \xi_{0}\right) & =\xi_{0}^{s-1} D_{1}\left(x, x_{0}, \xi\right) \\
& +\xi_{0}^{s-2} D_{2}\left(x, x_{0}, \xi\right)+\cdots+D_{s}\left(x, x_{0}, \xi\right)
\end{aligned}
$$


where $D_{j} \in \mathscr{B}\left([0, T]: S^{j}\right)$. Replacing $\mu_{j}\left(x, x_{0}, \xi\right) \quad(j=1, \cdots, s)$ for $\xi_{0}$ in (27) yields

$$
\begin{gathered}
\mu_{1}^{s-1} D_{1}\left(x, x_{0}, \xi\right)+\cdots+D_{s}\left(x, x_{0}, \xi\right)=D\left(x, x_{0}, \xi, \mu_{1}\right) \\
\vdots \\
\mu_{s}^{s-1} D_{1}\left(x, x_{0}, \xi\right)+\cdots+D_{s}\left(x, x_{0}, \xi\right)=D\left(x, x_{0}, \xi, \mu_{s}\right) .
\end{gathered}
$$

Denoting $(i, j)$-element of $D_{k}\left(x, x_{0}, \xi\right)$ by $d_{k}$ and $(i, j)$-element of $D(x$, $\left.x_{0}, \xi, \mu_{k}\right)$ by $\widehat{d}_{k}$, we simply have

$$
\begin{array}{cc}
\mu_{1}^{s-1} d_{1}+\cdots+d_{s}= & \hat{d}_{1} \\
\vdots & \vdots \\
\mu_{s}^{s-1} d_{1}+\cdots+d_{s}=\hat{\hat{d}}_{s} .
\end{array}
$$

We can solve this system for $d_{1}, \cdots, d_{s}$ since the coefficients make Van der Monde's determinant, so that we have $d_{k} \in \mathscr{B}\left([0, T]: S^{k-1}\right)$ since $\hat{d}_{k}$ $\in \mathscr{B}\left([0, T]: S^{s-1}\right)$. Therefore we can determine $D \in \widetilde{S}^{s-1}$, and also $C$ $\in \widetilde{S}^{N-s-1}$ from Remark in $\S 2$. The proof of Lemma 3.6 is completed.

(ii) Uniqueness

Let $u$ be a solution in $\mathscr{B}\left([0, T]: H^{l+N-2}\right)$ to the problem:

$$
\begin{aligned}
& M u=0 \\
& u(x, 0)=0 .
\end{aligned}
$$

Then $u$ satisfies

$$
\begin{aligned}
& (Q+\widetilde{\mathbf{X}}) M u=0 \\
& \left(D_{x_{0}}^{j} u\right)(x, 0)=0 \text { for } j=0,1, \cdots, N-1 .
\end{aligned}
$$

The uniqueness proof of (30) is thus reduced to that of (31).

Lemma 3. 7. Under the condition (I)' of Theorem 1 there exist symbols of pseudo-differential operators $\widetilde{C} \in \widetilde{S}^{N-s-1}, \widetilde{D} \in \widetilde{S}^{s-1}$ and $\widetilde{R}_{N-2}$ $\in \widetilde{S}^{N-2}$ so that

$$
(Q+\widetilde{\mathbf{X}}) M=(\alpha I+\widetilde{\mathbb{C}})(\beta I+\widetilde{\mathbb{D}})+\widetilde{\boldsymbol{R}}_{N-2} .
$$

Lemma 3. 7, Theorem 3.5 and (31) imply $u(x, t)=0$. Hence we proved the uniqueness of solution (1). 


$$
\sigma((Q+\widetilde{\mathbb{X}}) M) \equiv \operatorname{det}(P) I+\sum_{j=0}^{n} D_{\xi_{j}} Q \partial_{x_{j}} P-Q B+\widetilde{X} P,
$$

and

$$
\sigma((\alpha I+\widetilde{\boldsymbol{C}})(\beta I+\widetilde{\mathbb{D}})) \equiv \alpha \beta I+\sum_{j=0}^{n} D_{\xi_{j}} \alpha \partial_{x_{j}} \beta I+\alpha \widetilde{D}+\beta \widetilde{C}
$$

we want to determine symbols $\widetilde{C} \in \widetilde{S}^{x-s-1}, \widetilde{D} \in \widetilde{S}^{s-1}$ so that

$$
\sigma((\boldsymbol{Q}+\widetilde{\mathbf{X}}) \boldsymbol{M}) \equiv \sigma((\boldsymbol{\alpha} I+\widetilde{\mathbb{C}})(\beta I+\widetilde{\mathbb{D}}))
$$

or equivalently

$$
\sum_{j=0}^{n}\left(D_{\xi_{j}} Q \partial_{x_{j}} P-D_{\xi_{j}} \alpha \partial_{x_{j}} \beta I\right)+\widetilde{X} P-Q B=\alpha \widetilde{D}+\beta \widetilde{C}
$$

By virtue of the condition (I) this can be carried out in the same way as the proof of Lemma 3.6.

Finally we give the proof of Lemma 3.2 and 3.3.

Proof of Lemma 3.2. Since $\mathbb{P}_{m}$ is a strictly hyperbolic operator we have $P_{m}\left(x, x_{0}, \xi, \xi_{0}\right)=\prod_{j=1}^{m}\left(\xi_{0}-\lambda_{j}\left(x, x_{0}, \xi\right)\right)$ where $\left\{\lambda_{j}\right\}_{1 \leqq j \leqq m}$ are all real and distinct. We shall denote by $\partial_{j}$ the operator $D_{x_{0}}-\lambda_{j}\left(x, x_{0}, D_{x}\right) \quad(j$ $=1, \cdots, m)$. Then we can represent $\mathbb{P}_{m}$ in such a way that

$$
\mathbb{P}_{m}\left(x, x_{0}, D_{x}, D_{x_{0}}\right)=\partial_{m} \partial_{m-1} \cdots \partial_{1}+Q_{n-1}\left(x, x_{0}, D_{x}, D_{x_{0}}\right)
$$

where $Q_{m-1}\left(x, x_{0}, D_{x}, D_{x_{0}}\right)$ is a pseudo-differential operator with the symbol $Q_{m-1}\left(x, x_{0}, \xi, \xi_{0}\right) \in \widetilde{S}^{m-1}$. Hence we have

$$
\mathbb{P}=\mathbb{P}_{m} I+\mathbb{R}_{m-1}=\partial_{m} \partial_{m-1} \cdots \partial_{1} I+\widetilde{R}_{m-1}\left(x, x_{0}, D_{x}, D_{x_{0}}\right)
$$

where $\widetilde{\mathbb{R}}_{m-1}\left(x, x_{0}, D_{x}, D_{x_{0}}\right)$ is a pseudo differential operator with symbols in $\widetilde{S}^{m-1}$. Further we can represent $\widetilde{\mathbb{R}}_{m-1}\left(x, x_{0}, D_{x}, D_{x_{0}}\right)$ as follows:

$$
\widetilde{\boldsymbol{R}}_{m-1}\left(x, x_{0}, D_{x}, D_{x_{0}}\right)=\sum_{j=0}^{m-1} r_{m-j-1}\left(x, x_{0}, D_{x}\right) \partial_{j} \partial_{j-1} \cdots \partial_{1}
$$

where symbols of entries of $r_{i}\left(x, x_{0}, D_{x}\right)$ belong to $\mathcal{B}\left([0, T]: S^{i}\right) \quad(i=0$, $1, \cdots, m-1)$. Let us put

$$
\begin{gathered}
\left(u_{1}, \cdots, u_{N}, \partial_{1} u_{1}, \cdots, \partial_{1} u_{N}, \partial_{2} \partial_{1} u_{1}, \cdots, \partial_{m-1} \partial_{m-2} \cdots \partial_{1} u_{N}\right) \\
=\left(u_{1}^{0}, u_{2}^{0}, \cdots, u_{N}^{0}, u_{1}^{1}, \cdots, u_{N}^{1}, \cdots, u_{N}^{m-1}\right)
\end{gathered}
$$

and 


$$
U={ }^{t}\left(u_{1}^{0}, \cdots, u_{N}^{0}, \cdots, u_{N}^{m-1}\right) .
$$

By (37) and (38) the system of $N$ equations $\boldsymbol{P} u=f$ can be reduced to the following new system of $N m$ equations.

$$
D_{x_{0}} U=\boldsymbol{H}\left(x, x_{0}, D_{x}\right) U+F
$$

where

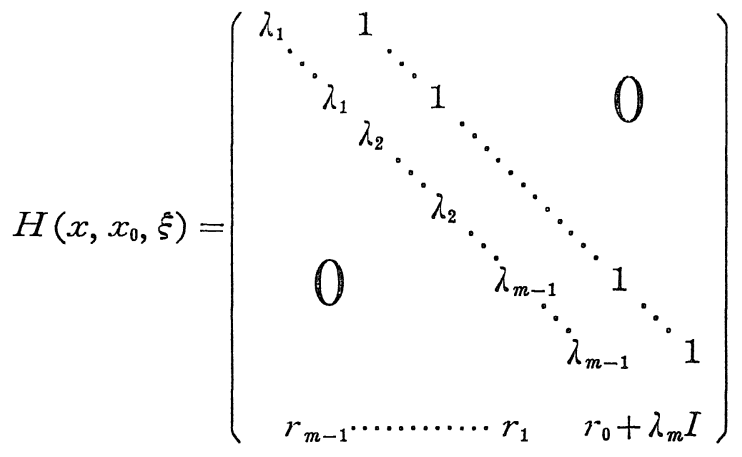

and $\quad F={ }^{t}\left(0,0, \cdots,{ }^{t} f\right)$. Put $\quad V={ }^{t}\left((\Lambda+1)^{m-1} u_{1}^{0}, \cdots,(\Lambda+1)^{m-1} u_{N}^{1}\right.$, $\left.(\Lambda+1)^{m-2} u_{1}^{1}, \cdots, u_{N}^{m-1}\right)$ in (39) where $\Lambda$ is a pseudo-differential operator with symbol $|\xi|$. Then we have

$$
D_{x_{0}} V=\boldsymbol{H}_{0}\left(x, x_{0}, D_{x}\right) \alpha V+\boldsymbol{B}\left(x, x_{0}, D_{x}\right) V+F
$$

where

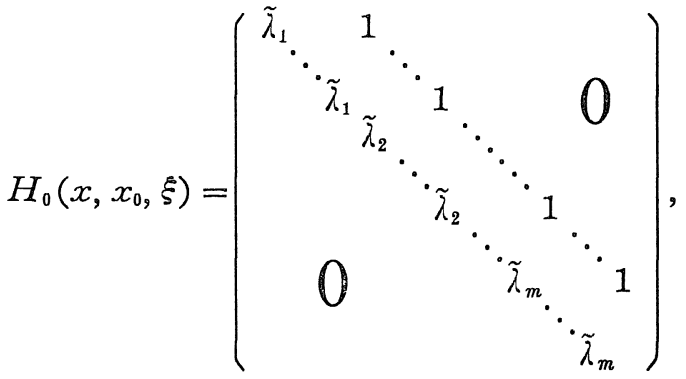

$$
\begin{aligned}
& \tilde{\lambda}_{j}\left(x, x_{0}, \xi\right)=\lambda_{j}\left(x, x_{0}, \xi\right)|\xi|^{-1}
\end{aligned}
$$

and $\boldsymbol{B}\left(x, x_{0}, D_{x}\right)$ is a pseudo-differential matrix operator with symbol in $\mathscr{B}\left([0, T]: S^{0}\right) . \quad$ As is well known, $\left(\begin{array}{cccc}\tilde{\lambda}_{1} & 1 & 0 \\ & \tilde{\lambda}_{2} & \ddots & \\ & & \ddots & 1 \\ 0 & & & \tilde{\lambda}_{n}\end{array}\right)$ has a diagonalizer $\left(a_{i j}\right)$ with $a_{i j}\left(x, x_{0}, \xi\right) \in \mathscr{B}\left([0, T]: S^{0}\right)$. This makes it possible to choose $L\left(x, x_{0}, \xi\right)=\left(a_{i j}\left(x, x_{0}, \xi\right) I\right)$ as a diagonalizer of $H_{0}\left(x, x_{0}, \xi\right)$ where $I$ is 
an $N \times N$ unit matrix. Finally putting $W=\mathbb{L}\left(x, x_{0}, D_{x}\right) V$ we get the following system

$$
D_{x_{0}} W=\widetilde{\boldsymbol{H}}_{0}\left(x, x_{0}, D_{x}\right) \Lambda W+\widetilde{\mathbb{B}}\left(x, x_{0}, D_{x}\right) W+\mathbb{L}\left(x, x_{0}, D_{x}\right) F .
$$

Here $\widetilde{\mathbb{H}_{0}}\left(x, x_{0}, \xi\right)=\left(\begin{array}{ccc}\tilde{\lambda}_{1} I & & 0 \\ & \ddots & \\ 0 & & \tilde{\lambda}_{m} I\end{array}\right)$ and $\widetilde{\mathbb{B}}\left(x, x_{0}, D_{x}\right)$ is a pseudo-differential operator with symbols in $\mathscr{B}\left([0, T]: S^{0}\right)$. Therefore we can obtain

$$
\begin{gathered}
\frac{d}{d x_{0}}\left\|D^{l} W\right\| \leqq \text { const. }\left\{\left\|D^{l} W\right\|+\left\|D^{l} F\right\|\right\} \\
\text { for } l=0,1, \cdots .
\end{gathered}
$$

This estimate and ellipticity of $\mathbb{L}$ imply (14), which proves our Lemma.

Proof of Lemma 3.3. Note that (4) implies

$$
D_{x_{0}}^{N} u=\sum_{j=0}^{N-1} a_{j}\left(x, x_{0}, D_{x}\right) D_{x_{0}}^{j} u+f
$$

with $a_{j}\left(x, x_{0}, \xi\right) \in \mathscr{B}\left([0, T]: S^{N-j}\right)$. We obtain (7) by differentiation of both sides of (44) with respect to $x_{0}$.

\section{§4. Proof of Theorem 2}

Consider the Cauchy problem

$$
\begin{aligned}
& \mathbb{M}(\mathbb{Q}+\mathbb{X}) u=f \\
& D_{x_{0}}^{j} u(x, 0)=\varphi_{j} \quad(j=0,1, \cdots, N-1) .
\end{aligned}
$$

Then we have

Proposition 4. 1. Under the condition (II) of Theorem 2, the energy inequalities

$$
\begin{gathered}
\left\|D^{l+N-2} u\right\| \leqq \text { const. }\left\{\sum_{j=0}^{N-1}\left\|\varphi_{j}\right\|_{N-j+m+l}+\sum_{j=0}^{m}\left\|D_{x_{0}}^{j} f(0)\right\|_{N-j+m+l}\right. \\
\left.+\int_{0}^{x_{0}}\left\|D^{l}\left(D_{x_{0}}^{m+1} f\left(x_{0}\right)\right)\right\| d x_{0}\right\}
\end{gathered}
$$

holds for $l=0,1, \cdots$, where $m=2(l-1)+N-s+m^{\prime}$ and $m^{\prime}$ depends only on operator $\mathbb{M}(\mathbb{Q}+\mathbb{X})$. 
In order to prove Proposition 4.1 we need the following three lemmas.

Lemma 4. 2. Under the condition (II) of Theorem 2 there exist symbols of pseudo-differential operators $C \in \widetilde{S}^{\mathrm{N-s}-1}$ and $D \in \widetilde{S}^{s-1}$ such that

$$
\boldsymbol{W}(\boldsymbol{Q}+\boldsymbol{X})=\left(\boldsymbol{\alpha} I+x_{0}^{-1} \boldsymbol{C}\right)\left(\boldsymbol{\beta} I+x_{0}^{-1} \mathbb{D}\right)+\mathfrak{S}_{N-2}
$$

where $\sigma\left(\mathbf{S}_{--2}\right)=\sum_{j=0}^{N-s-1} b_{j}\left(x, x_{0}, \xi, \xi_{0}\right) x_{0}^{-(j+2)}$ zeith $b_{j} \in \widetilde{S}^{N-j-2}$.

Lemma 4. 3. Let $\mathbb{P}=\mathbb{P}_{m} I+x_{0}^{-1} \boldsymbol{P}_{m-1}+\mathbb{P}_{m-2}+\cdots+\mathbb{P}_{0}$ be a pseudodifferential operator such that $\mathbb{P}_{n}$ is a strictly hyperbolic operator and each $\boldsymbol{P}_{j}(j=0,1, \cdots, m-1)$ is an $N \times N$ matrix pseudo-differential operator whose symbols belong to $\widetilde{S}^{j}$. Then we have the inequalities:

$$
\begin{gathered}
\frac{d}{d x_{0}}\left(\left\|D^{l+m-1} u\right\|^{2}\right) \leqq \text { const. }\left\{\left\|D^{l+m-1} u\right\|^{2}+\sum_{k=0}^{l}\left\|D^{l+m-1-k} u\right\|^{2} / x_{0}^{2 k+1}\right. \\
\left.\quad+x_{0}\left\|D^{l}(\boldsymbol{P} u)\right\|^{2}\right\} \\
\text { for } l=0,1, \cdots .
\end{gathered}
$$

Lemma 4. 4. If $u$ is a solution of (45), then we have

$$
\begin{gathered}
\left\|D_{x_{0}}^{N+l} u(x, 0)\right\|_{s} \leqq \text { const. }\left\{\sum_{j=0}^{N-1}\left\|\varphi_{j}\right\|_{N+l-j+s}+\sum_{i=1}^{l}\left\|\left(D^{i} f\right)(0)\right\|_{l-i+s}\right\} \\
\text { for } l=0,1, \cdots
\end{gathered}
$$

Proof of Proposition 4.1. To the proof we apply the same argument as in the proof of Proposition 3.1. Putting $S=\alpha I+x_{0}^{-1} \mathbb{C}$ and $\mathbb{R}$ $=\beta I+x_{0}^{-1} \mathbb{D}$ in (47) leads to

$$
\begin{aligned}
& \mathbb{R} u=v \\
& S v=f-S_{N-2} u .
\end{aligned}
$$

Let $w\left(x, x_{0}\right)$ be defined by $u\left(x, x_{0}\right)=x_{0} w\left(x, x_{0}\right)$. Then we have

$$
\begin{aligned}
& \mathbb{R} w=x_{0}^{-1}(v+\mathbb{E} w) \\
& \boldsymbol{S} v=f-\boldsymbol{S}_{N^{-2}}\left(x_{0} w\right)
\end{aligned}
$$


where $\sigma(\mathbb{E}) \in \widehat{S}^{s-1}$. Thus, the following inequalities hold by Lemma 4. 3 .

$$
\begin{aligned}
\frac{d}{d x_{0}}\left(\left\|D^{k+N-2-j} w\right\|^{2}\right) \leqq & \text { const. }\left\{\left\|D^{k+N-2-j} w\right\|^{2}\right. \\
& +\sum_{l^{\prime}=0}^{N-j-s-1+k}\left\|D^{k+N-2-j-l^{\prime}} w\right\|^{2} / x_{0}^{2 l^{\prime}+1} \\
& \left.+\sum_{l^{\prime}=0}^{N-j-s-1+h}\left\|D^{k+N^{j}-j-1-s-l^{\prime}} v\right\|^{2} / x_{0}^{2 l^{\prime}+1}\right\},
\end{aligned}
$$

$$
\begin{aligned}
\frac{d}{d x_{0}}\left(\left\|D^{k-j+N-s-1} v\right\|^{2}\right) \leqq & \text { const. }\left\{\left\|D^{k-j+N-s-1} v\right\|^{2}\right. \\
& +\sum_{l^{\prime}=0}^{k-j}\left\|D^{k+N-s-1-j-l^{\prime}} v\right\|^{2} / x_{0}^{2 l^{\prime}+1} \\
& \left.+x_{0}\left\|D^{k-j} f\right\|^{2}+x_{0}\left\|D^{k-j} \mathbb{S}_{N-2}\left(x_{0} w\right)\right\|^{2}\right\} .
\end{aligned}
$$

Since $\sigma\left(\mathbb{S}_{N-2}\right)=\sum_{j=0}^{N-s-1} b_{j} x_{0}^{-(j+2)}$ where $b_{j} \in \widetilde{S}^{N-2-j}$, Lemma 4.2 gives

$$
\begin{aligned}
\left\|D^{k-j} S_{N-2}\left(x_{0} w\right)\right\|^{2} & \leqq \text { const. }\left\{\sum_{l^{\prime}=0}^{k-j+N-s-1}\left\|D^{k-j+N-2-l^{\prime}}\left(x_{0} w\right)\right\|^{2} / x_{0}^{2\left(l^{\prime}+2\right)}\right\} \\
& \leqq \text { const. }\left\{\sum_{l^{\prime}=0}^{k-j+N-s-1}\left\|D^{k-j+N-2-l^{\prime}} w\right\|^{2} / x_{0}^{2\left(l^{\prime}+1\right)}\right\} .
\end{aligned}
$$

Combining (53) with (54) implies

$$
\begin{aligned}
\frac{d}{d x_{0}}\left(\left\|D^{N-s+k-j-1} v\right\|^{2}\right) \leqq & \text { const. }\left\{\left\|D^{N-s+k-j-1} v\right\|^{2}\right. \\
& +\sum_{l^{\prime}=0}^{k-j}\left\|D^{N-s+k-j-1-l^{\prime}} v\right\|^{2} / x_{0}^{2 l^{\prime}+1}+x_{0}\left\|D^{k-1} f\right\|^{2} \\
& \left.+\sum_{l^{\prime}=0}^{k-j+N-s-1}\left\|D^{k-j+N-2-l^{\prime}} w\right\|^{2} / x_{0}^{2 l^{\prime}+1}\right\} .
\end{aligned}
$$

Hence we obtain, by summing (52) and (55),

$$
\begin{gathered}
\frac{d}{d x_{0}}\left(\left\|D^{k+N-2-j} w\right\|^{2}+\left\|D^{N-s+k-j-1} v\right\|^{2}\right) \leqq \text { const. }\left\{\left\|D^{k+N-2-j} w\right\|^{2}\right. \\
+\left\|D^{N-s+k-j-1} v\right\|^{2}+\sum_{l^{\prime}=0}^{k-j+N-s-1}\left(\left\|D^{k-N-j-1-s-l^{\prime}} v\right\|^{2}\right. \\
\left.\left.+\left\|D^{k-j+N-2-l^{\prime}} w\right\|^{2}\right) / x_{0}^{2 l^{\prime}+1}+x_{0}\left\|D^{k-j} f\right\|^{2}\right\} .
\end{gathered}
$$

Now we define a function $\Phi$ of $x_{0}$ by 


$$
\Phi\left(x_{0}\right)=\sum_{k=0}^{l} \sum_{j=0}^{k+N-s-1}\left(\left\|D^{k+N-2-j} w\right\|^{2}+\left\|D^{N-s+k-j-1} v\right\|^{2}\right) / x_{0}^{2 j+1} .
$$

Then from (56) it follows that

$$
\begin{aligned}
\frac{d\left(x_{0} \Phi\left(x_{0}\right)\right)}{d x_{0}} & \leqq \gamma x_{0} \Phi\left(x_{0}\right)+c \Phi\left(x_{0}\right)+\text { const. } \sum_{k=0}^{l} \sum_{j=0}^{k+N-s-1} x_{0}^{2}\left\|D^{k-j} f\right\|^{2} / x_{0}^{2 j+1} \\
& \leqq \gamma x_{0} \Phi\left(x_{0}\right)+c \Phi\left(x_{0}\right)+\text { const. } x_{0}^{-2(N-s-1)+1} \sum_{k=0}^{b}\left\|D^{k} f\right\|^{2} / x_{0}^{2 k}
\end{aligned}
$$

where $\gamma$ and $c$ are positive constants depending only on equation (45). Hence we have

$$
\begin{aligned}
\frac{d}{d x_{0}}\left(x_{0}^{-c+1}\right. & \left.\exp \left(-\gamma x_{0}\right) \Phi\left(x_{0}\right)\right) \\
& \leqq \text { const. } x_{0}^{-c} \exp \left(-\gamma x_{0}\right) x_{0}^{-2(N-s-1)+1} \sum_{k=0}^{l}\left\|D^{k} f\right\|^{2} / x_{0}^{2 k}
\end{aligned}
$$

To make the value $\left[x_{0}^{-c+1} \exp \left(-\gamma x_{0}\right) \Phi\left(x_{0}\right)\right]_{x_{0}=0}$ finite when integrating both sides of (58), we redefine a formula $\Phi\left(x_{0}\right)$ by replacing

$$
z\left(x, x_{0}\right)=u\left(x, x_{0}\right)-\sum_{j=0}^{N+m}\left(i x_{0}\right)^{j} D_{x_{0}}^{j} u(x, 0) / j !
$$

for $\iota=x_{0} w$ in (57). Here $m$ is a suitable integer specified later. Then $z\left(x, x_{0}\right)$ satisfies

$$
\begin{aligned}
& T \approx=g \\
& D_{x_{0}}^{k} z(x, 0)=0 \text { for } k \leqq N+m
\end{aligned}
$$

where $\boldsymbol{T}=\boldsymbol{M}(\boldsymbol{Q}+\boldsymbol{X})$ and $g=f-\boldsymbol{T}\left(\sum_{j=0}^{N+m}\left(i x_{0}\right)^{j} D_{x_{0}}^{j} u(x, 0) / j !\right)$. Since $D_{x_{0}}^{k} g(x, 0)=0$ for $k \leqq m$, we have $g\left(x, x_{0}\right)=\frac{1}{m !} \int_{0}^{x_{0}}\left(x_{0}-s\right)^{m}\left(i D_{s}\right)^{m+1} g(x, s) d s$ and then

$$
\left\|D^{l} g\left(x_{0}\right)\right\|^{2} \leqq \text { const. } x_{0}^{2(m-l)+1} \int_{0}^{x_{0}}\left\|D^{l} D_{s}^{m+1} g(x, s)\right\|^{2} d s \text {. }
$$

Now from the estimate (58) for $\approx\left(x, x_{0}\right)=x_{0} \widetilde{w}\left(x, x_{0}\right)$ and (60) we immediately have

$$
\begin{array}{ll}
D_{x_{0}}^{k} \widetilde{\widetilde{v}}(x, 0)=0 & \text { for } k \leqq N+m-1, \\
D_{x_{0}}^{k} \widetilde{\mathcal{v}}(x, 0)=0 & \text { for } k \leqq N+m-s-1 .
\end{array}
$$

Thus for our purpose we must choose $m$ so that 


$$
\begin{aligned}
& 2(N+m-1) \geqq 2(k+N-2-j)+(2 j+1)+(c-1), \\
& 2(N+m-s-1) \geqq 2(N-s+k-j-1)+(2 j+1)+(c-1) \\
& \quad \text { for } j=0,1, \cdots, k+N-s-1, k=0,1, \cdots, l .
\end{aligned}
$$

Therefore we may take $m>1+\frac{1}{2} c$, which implies

$$
\left[x_{0}^{-c+1} \exp \left(-\gamma x_{0}\right) \Phi\left(x_{0}\right)\right]_{x_{0}=0}=0 .
$$

Carrying out the integration over $\left[0, x_{0}\right]$ in (58) we obtain

$$
\begin{aligned}
\left\|D^{l+N-2} \widetilde{w}\left(x_{0}\right)\right\|^{2} & \leqq \text { const. } x_{0}^{c} \exp \left(\gamma x_{0}\right) \int_{0}^{x_{0}} s^{-2(N-s-1)-c+1} \sum_{k=0}^{l}\left\|D^{k} g\right\|^{2} / s^{2 k} d s \\
& \leqq \text { const. } x_{0}^{c} \exp \left(\gamma x_{0}\right) \int_{0}^{x_{0}} s^{-2(N-s-1)-c+1-2 l}\left\|D^{l} g\right\|^{2} d s,
\end{aligned}
$$

and further, taking (61) into consideration we have

$$
\begin{gathered}
\left\|D^{\imath+N-2} \widetilde{w}\left(x_{0}\right)\right\|^{2} \leqq \text { const. } \int_{0}^{x_{0}} s^{-2(N-s-1)-c+1-2 l+2(m-l)+1} d s \\
\times \int_{0}^{x_{0}}\left\|D^{l} D_{s}^{m+1} g(s)\right\|^{2} d s .
\end{gathered}
$$

Here we choose $m$ so that $-2(N-s-1)-c+1-2 l+2(m-l)+1>-1$ i.e. $m>2(l-1)+N-s+\frac{c-1}{2}$. Then we have

$$
\left\|D^{l+N-2} \widetilde{\mathfrak{w}}\left(x_{0}\right)\right\|^{2} \leqq \text { const. } \int_{0}^{x_{0}}\left\|D^{l} D_{s}^{m+1} g(s)\right\|^{2} d s
$$

If $m>2(l-1)+N-s+\frac{c}{2}+1$ then $m$ satisfies both $m>l+\frac{c}{2}$ and $m>2(l-1)+N-s+\frac{c-1}{2}$. Consequently we put in (59) $m=2(l-1)$ $+N-s+m^{\prime}$ where $m^{\prime}=\left[\frac{c}{2}\right]+2$. Thus we have easily from (64) for $z\left(x, x_{0}\right)=x_{0} \widetilde{w}\left(x, x_{0}\right)$

$$
\left\|D^{l+N-2} z\left(x, x_{0}\right)\right\|^{2} \leqq \text { const. } \int_{0}^{x_{0}}\left\|D^{l} D_{s}^{m+1} g(s)\right\|^{2} d s .
$$

Since $u\left(x, x_{0}\right)=z\left(x, x_{0}\right)+\sum_{j=0}^{N+m}\left(i x_{0}\right)^{j} D_{x_{0}}^{j} u(x, 0) / j$ ! we finally obtain

$$
\begin{aligned}
\left\|D^{l+N-2} u\left(x, x_{0}\right)\right\|^{2} \leqq & \text { const. }\left\{\sum_{j=0}^{N+m}\left\|D_{x_{0}}^{j} u(x, 0)\right\|_{l+N-2}^{2}\right. \\
& \left.+\int_{0}^{x_{0}}\left\|D^{l} D_{s}^{m+1} g(s)\right\|^{2} d s\right\}
\end{aligned}
$$


which, combined with Lemma 4.4, yields the estimate (46). Therefore we have completed the proof of Proposition 4.1.

Now we shall return to prove Lemmas $4.2,4.3,4.4$.

Proof of Lemma 4.2. We shall calculate the symbols of $\mathbb{M}(\boldsymbol{Q}+\boldsymbol{X})$ and $\left(\alpha I+x_{0}^{-1} \mathcal{C}\right)\left(\beta I+x_{0}^{-1} \mathbb{D}\right)$

$$
\begin{gathered}
\sigma(\mathbb{M}(\boldsymbol{Q}+\boldsymbol{X}))=\operatorname{det}(P) I+\sum_{j=0}^{n} D_{\xi} P \partial_{x_{j}} Q-B Q+P X, \\
\begin{array}{c}
\sigma\left(\left(\alpha I+x_{0}^{-1} \mathbb{C}\right)\left(\beta I+x_{0}^{-1} \mathbb{D}\right)\right)=\alpha \beta I+\sum_{j=0}^{n} D_{\xi_{j}} \alpha \partial_{x_{j}} \beta I \\
+x_{0}^{-1}(\alpha D+\beta C)+R_{N-2}
\end{array}
\end{gathered}
$$

where

$$
\begin{gathered}
R_{N-2}=x_{0}^{-2} C D+\sum_{j=0}^{n}\left\{D_{\xi} \alpha \partial_{x_{j}}\left(x_{0}^{-1} D\right)+x_{0}^{-1} D_{\xi_{j}} C \partial_{x_{j}}\left(\beta I+x_{0}^{-1} D\right)\right\} \\
\sum_{|r| \gtrless^{2}} \frac{1}{\gamma !} D_{\xi}^{r}\left(\alpha I+x_{0}^{-1} C\right) \partial_{x}^{r}\left(\beta I+x_{0}^{-1} D\right) .
\end{gathered}
$$

We take $C \in \widetilde{S}^{N-s-1}$ and $D \in \widetilde{S}^{s-1}$ so that

$$
\sum_{j=0}^{n}\left(D_{\xi j} P \partial_{x_{j}} Q-D_{\xi_{j}} \alpha \partial_{x_{j}} \beta I\right)+P X-B Q=x_{0}^{-1}(\alpha D+\beta C),
$$

which can be obtained by the similar way to the proof of Lemma 3.6. Let $\left.S_{N-2}=\sigma(\mathbb{Q}+\mathbb{Q})\right)-\sigma\left(\left(\alpha I+x_{0}^{-1} \boldsymbol{C}\right)\left(\boldsymbol{\beta} I+x_{0}^{-1} \mathbb{D}\right)\right)$. Then

$$
\begin{aligned}
-S_{\mathrm{v}-2}=x_{0}^{-2} C D & +\sum_{j=0}^{n}\left\{D_{\xi_{j}} \alpha \partial_{x_{j}}\left(x_{0}^{-1} D\right)+x_{0}^{-1} D_{\xi_{j}} C \partial_{x_{j}}\left(\beta I+x_{0}^{-1} D\right)\right\} \\
& +\sum_{|r| \geqq 2} D_{\xi}^{r}\left(\alpha I+x_{0}^{-1} C\right) \partial_{x}^{r}\left(\beta I+x_{0}^{-1} D\right) .
\end{aligned}
$$

The last term of the right hand side is

$$
\begin{gathered}
\sum_{|\gamma| \geqslant 2} \frac{1}{\gamma !} D_{\xi}^{r} \alpha \partial_{x}^{r}\left(\beta I+x_{0}^{-1} D\right)+\sum_{|\gamma| \geq 2} \frac{1}{\gamma !} x_{0}^{-1} D_{\xi}^{r} C \partial_{x}^{r}\left(\beta I+x_{0}^{-1} D\right) \\
=I+I I .
\end{gathered}
$$

Since the order of $\alpha$ is $N-s$, all the elements of $I$ vanish when $\gamma_{0}$ $>N-s$. Also since the order of $C$ is $N-s-1$, all the elements of $I I$ vanish when $r_{0}>N-s-1$. Thus we have 


$$
S_{N-2}=\sum_{j=0}^{N-s-1} h_{j}\left(x, x_{0}, \tilde{\xi}, \tilde{\xi}_{0}\right) x_{0}^{-(j+2)} \quad \text { with } b_{j} \in \widetilde{S}^{N-2-j}
$$

Proof of Lemma 4.3. We remark the same method as the proof of Lemma 3.2 reduces the system of $N$ equations $P u=f$ to the system of $N m$ equations as follows.

$$
D_{x_{0}} W=\widetilde{\boldsymbol{H}}_{0}\left(x, x_{0}, D_{x}\right) d W+x_{0}^{-1} \widetilde{\mathbb{B}}\left(x, x_{0}, D_{x}\right) W+\mathbb{L}\left(x, x_{0}, D_{x}\right) F
$$

where $\widetilde{\boldsymbol{H}}_{0}, \mathbb{Z}$ are the same pseudo-differential operators in Lemma 3.2 and $\widetilde{\mathbb{B}}$ is a pseudo-differential operators with symbols in $\mathcal{B}\left([0, T]: S^{0}\right)$. Then (43) and the identity

$$
D_{x_{0}}^{\alpha_{0}} D_{x}^{\alpha}\left(x_{0}^{-1} \widetilde{\mathbb{B}} W\right)=\sum_{j \leq \alpha_{0}} x_{0}^{-(j+1)}(i)^{j}\left(\begin{array}{c}
\alpha_{0} \\
j
\end{array}\right) D_{x_{0}}^{\alpha_{0}-j} D_{x}^{\alpha}(\widetilde{B} W)
$$

yield

$$
\begin{aligned}
\frac{d}{d x_{0}}\left(\left\|D^{l} W\right\|^{2}\right) \leqq & \text { const. }\left\{\sum_{j=0}^{l} x_{0}^{-(2 j+1)}\left\|D^{l-j} W\right\|^{2}+\left\|D^{l} W\right\|^{2}\right. \\
& \left.+\left|\left(D^{l} W, D^{l}(\mathbb{L} F)\right)\right|\right\} .
\end{aligned}
$$

Since Schwarz' inequality gives

$$
\begin{aligned}
& \left|\left(D^{l} W, D^{l}(\mathbb{L} F)\right)\right|=\left|\left(x_{0}^{-\frac{1}{2}} D^{l} W, x_{0}^{\frac{1}{2}} D^{l}(\mathbb{L} F)\right)\right| \\
& \leqq \text { const. }\left\{x_{0}^{-1}\left\|D^{l} W\right\|^{2}+x_{0}\left\|D^{l} F\right\|^{2}\right\}
\end{aligned}
$$

we have

$$
\frac{d}{d x_{0}}\left(\left\|D^{l} W\right\|^{2}\right) \leqq \text { const. }\left\{\sum_{j=0}^{l} x_{0}^{-(2 j+1)}\left\|D^{l-j} W\right\|^{2}+\left\|D^{l} W\right\|+x_{0}\left\|D^{l} F\right\|\right\}
$$

Finally combining this estimate (71) with ellipticity of $\mathbb{R}$ implies (48).

Proof of Lemma 4. 4. The proof is done by the same method as in the case of Lemma 3.3.

Proposition 4. 5. Let $\mathbb{T}^{*}$ be an adjoint operator of $\mathbb{T}$. Then there exist pseudo-differential operators $\widetilde{\mathbb{C}}, \widetilde{\mathbb{D}}$ reith symbols $\sigma(\mathbb{C})$ $\in \widetilde{S}^{N-s-1}, \sigma(\mathbb{D}) \in \widetilde{S}^{s-1}$ such that

$$
T^{*}=\left(\beta I+x_{0}^{-1} \widetilde{\mathbb{D}}\right)\left(\alpha I+x_{0}^{-1} \widetilde{\boldsymbol{C}}\right)+\widetilde{S}_{N-2}
$$


where $\sigma\left(\boldsymbol{S}_{N-2}\right)=\sum_{j=0}^{N-s-1} b_{j}\left(x, x_{0}, \xi, \xi_{0}\right) x_{0}^{-(j+2)}$ with $b_{j} \in \widetilde{S}^{N-j-2}$.

The proof of Proposition 4.5 can be done along the same way as the proofs of Proposition 3.4 and Lemma 4.2.

Proposition 4. 6. Let $v \in \mathscr{B}\left([0, T]: H^{l+N-2}\right)$ be a solution of the Cauchy problem:

$$
\begin{aligned}
& \boldsymbol{T}^{*} v=h(x, t) \in \mathcal{B}\left([0, T]: H^{2 m+l}\right) \quad \text { in } \mathbb{R}^{n} \times[0, T] \\
& D_{t}^{j} v(x, T)=\varphi_{j}(x) \in H^{N-j+l-1+m} \quad(j=0,1, \cdots, N-1) .
\end{aligned}
$$

Then we have the energy inequalities:

$$
\begin{aligned}
\int_{0}^{T}\left\|D^{l+N-2} \mathscr{v}\right\|^{2} d t \leqq & \text { const. }\left\{\sum_{j=0}^{N-1}\left\|\varphi_{j}\right\|_{N-j+l-1+m}^{2}+\left\|D^{2 l-3} h\right\|_{m-l+2}^{2}\right. \\
& \left.+\int_{0}^{T}\left\|D^{l+m-2} h(t)\right\|_{m-l+2}^{2} d t\right\} \\
\text { for } l= & 0,1, \cdots
\end{aligned}
$$

where $m=(l+s) m^{\prime}+1$ and $m^{\prime}$ is a non-negative integer depending only the operator $T^{*}$.

Proof. We can show the inequalities (74) hold using Proposition 4.5 by the same method of Ohya [1].

From Propositoon 4. 1 and Proposition 4.6 we can obtain the following theorem by the method of Nirenberg [10].

Theorem 4. 7. Under the condition (II) of Theorem 2 the Cauchy problem (45) is rell-posed in the space $H^{-\infty}$ for the future $0 \leqq x_{0}$ $\leqq T$.

To the Cauchy problem

$$
\begin{aligned}
& (\mathbb{Q}+\mathbf{X}) M u=f \\
& \left(D_{x_{0}}^{j} u\right)(x, 0)=\varphi_{;} \quad(j=0,1, \cdots, N-1)
\end{aligned}
$$

quite the same method of proof as Theorem 4.7 can be applied, as is 
easily seen. Therefore we have

Theorem 4. 8. Under the condition (II)' of Theorem 2 the Cauchy problem for (75) is rell-posed in the space $H^{-\infty}$ for the future $0 \leqq x_{0} \leqq T$.

Now we call complete the proof of Theorem 2, by using Theorem 4. 7 for the existence theorem for (1) and Theorem 4.8 for the uniqueness theorem for (1).

\section{References}

[1] Ohya, Y., Le Problème de Cauchy à Caractéristiques Multiples, to appear.

[2] Menikoff, A., The Cauchy problem for weakly hyperbolic equations, Amer. $J$. Math., 97 (1975), 548-558.

[3] Oleinik, O., On the Cauchy problem for weakly hyperbolic equations, Comm. Pure Appl. Math., 23 (1970), 569-586.

[4] Petkov, V., On the Cauchy Problem for first order hyperbolic systems with multiple characteristics, Soviet Math. Dokl., 14 (1973), 534-537.

[5] Yamahara, H., On the Cauchy problem for weakly hyperbolic systems, Proc. Japan Acad., 52 (1976), 167-170.

[6] Demay, Y., Le problème de Cauchy pour les systèms hyperboliques à caractéristiques double, C. R. Acad. Sci. Paris, 278 (1974), 771-773.

[7] Mizohata, S. and Ohya, Y., Sur la condition de E. E. Levi concernant des équations hyperboliques, Publ. RIMS Kyoto Univ., Ser. A 4 (1968), 511-526.

[8] Flaschka, H. and Strang, G., The correctness of the Cauchy problem, Adv. in Math., 6 (1971), 347-379.

[9] Chazarain, J., Opérateurs hyperboliques a caractéristiques de multiplicité constante, Ann. Inst. Fourier (Grenoble), 24 (1971), 173-202.

[10] Nirenberg, L., Pseudo-differential operators in Global Analysis, Proc. Sympos. Pure Math., 16 (1970), 149-167. 
